PROCEEDINGS OF THE

AMERICAN MATHEMATICAL SOCIETY

Volume 125, Number 12, December 1997, Pages 3489-3500

S 0002-9939(97)04037-9

\title{
THE HAAR MEASURE ON FINITE QUANTUM GROUPS
}

\author{
A. VAN DAELE
}

(Communicated by Palle E. T. Jorgensen)

\begin{abstract}
By a finite quantum group, we will mean in this paper a finitedimensional Hopf algebra. A left Haar measure on such a quantum group is a linear functional satisfying a certain invariance property. In the theory of Hopf algebras, this is usually called an integral. It is well-known that, for a finite quantum group, there always exists a unique left Haar measure. This result can be found in standard works on Hopf algebras.

In this paper we give a direct proof of the existence and uniqueness of the left Haar measure on a finite quantum group. We introduce the notion of a faithful functional and we show that the Haar measure is faithful. We consider the special case where the underlying algebra is a ${ }^{*}$-algebra with a faithful positive linear functional. Then the left and right Haar measures coincide. Finally, we treat an example of a root of unity algebra. It is an example of a finite quantum group where the left and right Haar measures are different.

This note does not contain many new results but the treatment of the finite-dimensional case is very concise and instructive.
\end{abstract}

\section{INTRODUCTION}

Let $A$ be an algebra over $\mathbb{C}$ with identity. A comultiplication is a homomorphism $\Delta$ from $A$ to $A \otimes A$ that satisfies the coassociativity law $(\Delta \otimes \iota) \Delta=(\iota \otimes \Delta) \Delta$ where $\iota$ is the identity map. A counit is a homomorphism $\epsilon: A \rightarrow \mathbb{C}$ such that $(\epsilon \otimes \iota) \Delta=(\iota \otimes \epsilon) \Delta=\iota$. An antipode is a anti-homomorphism $S: A \rightarrow A$ such that $m(S \otimes \iota) \Delta(a)=m(\iota \otimes S) \Delta(a)=\epsilon(a) 1$ for all $a \in A$ where $m: A \otimes A \rightarrow A$ is the linear map defined by $m(a \otimes b)=a b$ when $a, b \in A$. If the counit and the antipode exist, then they are unique. A pair $(A, \Delta)$ where $\Delta$ is a comultiplication on $A$ is called a Hopf algebra if such a counit and an antipode exist. We refer to the books of Abe [1] and Sweedler [6] for results on Hopf algebras. See also the survey paper on dual pairs of Hopf *-algebras [7].

If $A$ is a Hopf algebra, the space $A^{\prime}$ of linear functionals is again an algebra with identity. The product is given by $(f g)(a)=(f \otimes g) \Delta(a)$ and $\epsilon$ is the identity. If $A$ is finite-dimensional, $A^{\prime}$ is again a Hopf algebra with comultiplication $\Delta: A^{\prime} \rightarrow A^{\prime} \otimes A^{\prime}$ defined by $(\Delta f)(a \otimes b)=f(a b)$. Here we identify $A^{\prime} \otimes A^{\prime}$ with $(A \otimes A)^{\prime}$. This is possible because $A$ is finite-dimensional. The counit on $A^{\prime}$ is given by $\epsilon(f)=f(1)$ and the antipode on $A^{\prime}$ is defined by $(S f)(a)=f(S a)$ for all $a \in A$.

If $G$ is a finite group and $A$ the algebra of functions $f: G \rightarrow \mathbb{C}$ with pointwise operations, then $A$ is a Hopf algebra if we define $\Delta$ by $(\Delta f)(p, q)=f(p q)$ where we identify $A \otimes A$ with the complex functions on $G \times G$. The counit is given by

Received by the editors March 18, 1996 and, in revised form, July 8, 1996.

1991 Mathematics Subject Classification. Primary 16W30. 
$\epsilon(f)=f(e)$ where $e$ is the identity in $G$ and the antipode is given by $(S f)(p)=$ $f\left(p^{-1}\right)$. The dual $A^{\prime}$ of $A$ can be identified with the space of complex functions on $G$ but now the product in $A^{\prime}$ is the convolution product. So $A^{\prime}$ is the usual group algebra of $G$ over $\mathbb{C}$. If we denote by $\lambda_{p}$ the image of the element $p \in G$ in $A^{\prime}$, then $\Delta\left(\lambda_{p}\right)=\lambda_{p} \otimes \lambda_{p}, \epsilon\left(\lambda_{p}\right)=1$ and $S\left(\lambda_{p}\right)=\lambda_{p^{-1}}$ for all $p$.

In the example above we can consider the linear map $\varphi: A \rightarrow \mathbb{C}$ given by $\varphi(f)=\sum_{p \in G} f(p)$. Because $\sum_{p \in G} f(q p)=\sum_{p \in G} f(p)$ for all $q \in G$ we have

$$
(\iota \otimes \varphi) \Delta(f)=\varphi(f) 1 \text {. }
$$

In the general case, a (non-zero) linear map $\varphi: A \rightarrow \mathbb{C}$ is called a left invariant Haar functional if $(\iota \otimes \varphi) \Delta(a)=\varphi(a) 1$ for all $a \in A$. Similarly, a linear map $\psi: A \rightarrow \mathbb{C}$ is called a right invariant Haar functional if $(\psi \otimes \iota) \Delta(a)=\psi(a) 1$ for all $a \in A$. Also for the group algebra we have a Haar functional. It is given by $\varphi\left(\lambda_{p}\right)=0$ if $p \neq e$ and $\varphi\left(\lambda_{e}\right)=1$.

In [1] and [6] it is shown that such a left invariant Haar functional exists in the case of a finite-dimensional Hopf algebra and that it is unique in any case. Of course, the same is true for right invariant Haar functionals.

In this paper we give a short direct proof of the existence and uniqueness of the left Haar functional $\varphi$ on a finite-dimensional Hopf algebra. We prove that $\varphi$ is faithful in the sense that $\varphi(b a)=0$ for all $b$ implies $a=0$. The argument can even be simplified a little if we have given a faithful functional. This applies to the case where $A$ is a finite-dimensional $\mathrm{C}^{*}$-algebra. This case can be characterized by the fact that $A$ is a ${ }^{*}$-algebra with a faithful positive linear functional. Then, we also give an argument for the positivity of the Haar functional and we use it to show that $A^{\prime}$ is again a $\mathrm{C}^{*}$-algebra. In this case, the left and right Haar functionals coincide. They are traces. We also give a short argument for the fact that here $S^{2}=\iota$. We explain the relation with the formula for the Haar functional in [8] on discrete quantum groups. See also [2], [3] and [9] for this finite-dimensional case.

In the last section of this paper we treat an example of a finite-dimensional Hopf algebra with different left and right Haar functionals. We obtain explicit formulas for these functionals and we also illustrate some other aspects of the theory here.

We will work with the common index notation in Hopf algebras. So we use

$$
\begin{aligned}
\Delta(a) & =\sum_{(a)} a_{(1)} \otimes a_{(2)}, \\
(\Delta \otimes \iota) \Delta(a) & =\sum_{(a)} a_{(1)} \otimes a_{(2)} \otimes a_{(3)} .
\end{aligned}
$$

Formulas like $m(\iota \otimes S) \Delta(a)=\epsilon(a) 1$ and $(\epsilon \otimes \iota) \Delta(a)=a$ e.g. become

$$
\begin{gathered}
\sum_{(a)} a_{(1)} S a_{(2)}=\epsilon(a) 1, \\
\sum_{(a)} \epsilon\left(a_{(1)}\right) a_{(2)}=a .
\end{gathered}
$$

The reader is assumed to have (or to get) some acquaintance with these formulas.

We will sometimes write $\langle f, a\rangle$ for $f(a)$ when $f \in A^{\prime}$ and $a \in A$ to avoid the use of too many brackets. This will increase the readability of the formulas. For the same reason we leave out brackets in $S a b$ when we mean $(S(a)) b$. 


\section{The HaAR Functional on finite-dimensional Hopf algebras}

Let $A$ be a finite-dimensional Hopf algebra over $\mathbb{C}$. We will give a short and direct proof of the existence, uniqueness and faithfulness (to be defined later) of the left and right Haar functionals on $A$. In fact, we will construct these functionals first on the dual Hopf algebra $A^{\prime}$. Observe that the left Haar functional on $A^{\prime}$ is given by a non-zero element $h$ in $A$ such that $a h=\epsilon(a) h$ for all $a \in A$.

1.1. Proposition. There exists a non-zero element $h$ in $A$ such that ah $=\epsilon(a) h$ for all $a \in A$.

Proof. Let $\left(a_{i}\right)$ be a basis for $A$ and let $\left(f_{i}\right)$ be the dual basis for $A^{\prime}$. For any $b \in A$, define $h \in A$ by

$$
h=\sum_{i,\left(a_{i}\right)}\left\langle f_{i}, S^{2} a_{i(2)} b\right\rangle a_{i(1)}
$$

We will show first that $a h=\epsilon(a) h$ for all $a \in A$.

Take $a \in A$. Then we get

$$
\begin{aligned}
\epsilon(a) h & =\sum_{i,\left(a_{i}\right),(a)}\left\langle f_{i}, a_{(1)} S a_{(2)} S^{2} a_{i(2)} b\right\rangle a_{i(1)} \\
& =\sum_{i, j,\left(a_{i}\right),(a)}\left\langle f_{i}, a_{(1)} a_{j}\right\rangle\left\langle f_{j}, S a_{(2)} S^{2} a_{i(2)} b\right\rangle a_{i(1)} \\
& =\sum_{j,\left(a_{j}\right),(a)}\left\langle f_{j}, S a_{(3)} S^{2}\left(a_{(2)} a_{j(2)}\right) b\right\rangle a_{(1)} a_{j(1)} \\
& =\sum_{j,\left(a_{j}\right)}\left\langle f_{j}, S^{2} a_{j(2)} b\right\rangle a a_{j(1)} \\
& =a h .
\end{aligned}
$$

Now let $h_{j}$ denote the element associated with $a_{j}$. Then

$$
\begin{aligned}
\sum_{j}\left\langle f_{j}, S h_{j}\right\rangle & =\sum_{i, j,\left(a_{i}\right)}\left\langle f_{i}, S^{2} a_{i(2)} a_{j}\right\rangle\left\langle f_{j}, S a_{i(1)}\right\rangle \\
& =\sum_{i,\left(a_{i}\right)}\left\langle f_{i}, S^{2} a_{i(2)} S a_{i(1)}\right\rangle \\
& =\sum_{i} \epsilon\left(a_{i}\right)\left\langle f_{i}, 1\right\rangle \\
& =\epsilon(1)=1 .
\end{aligned}
$$

Hence, some $h_{j}$ must be non-zero. This proves the result.

This proposition proves the existence of a left Haar functional on $A^{\prime}$. By duality, we also get the existence of a left Haar functional on $A$. Uniqueness will follow easily from the next result.

1.2. Lemma. Let $h$ be a non-zero element in $A$ such that ah $=\epsilon(a) h$ for all $a \in A$. Then the map $\omega \rightarrow(\iota \otimes \omega) \Delta(h)$ from $A^{\prime}$ to $A$ is bijective. 
Proof. It is enough to show that this map is injective. So assume $\omega \in A^{\prime}$ and $(\iota \otimes \omega) \Delta(h)=0$. For any element $a \in A$ we have

$$
\begin{aligned}
(1 \otimes a) \Delta(h) & =\sum_{(a)}\left(S a_{(1)} a_{(2)} \otimes a_{(3)}\right) \Delta(h) \\
& =\sum_{(a)}\left(S a_{(1)} \otimes 1\right) \Delta\left(a_{(2)} h\right) \\
& =(S a \otimes 1) \Delta(h) .
\end{aligned}
$$

So $(\iota \otimes \omega)((1 \otimes a) \Delta(h))=S a((\iota \otimes \omega) \Delta(h))=0$. Then, if we apply $\Delta$, and replace $a$ by $a S b$, we find

$$
\sum_{(h)} \omega\left(a S b h_{(3)}\right) h_{(1)} \otimes h_{(2)}=0
$$

for all $a, b \in A$. If we replace $b$ by $a_{i}$, apply $\iota \otimes f_{i}$ (where $\left(a_{i}\right)$ and $\left(f_{i}\right)$ are a basis and a dual basis as before) and if we sum over $i$, we find

$$
\sum_{(h)} \omega\left(a S h_{(2)} h_{(3)}\right) h_{(1)}=0 .
$$

Then, $\omega(a) h=0$ for all $a$ and because $h \neq 0$, we have $\omega=0$.

If now $\varphi$ is a left Haar functional, then $(\iota \otimes \varphi) \Delta(h)=\varphi(h) 1$. By the above result, $\varphi(h) \neq 0$. So we can normalise $\varphi$ such that $\varphi(h)=1$. Again by the above result, $\varphi$ is unique. So we get the following.

1.3. Theorem ([1], [6]). There is a unique (up to a scalar) left invariant Haar functional on any finite-dimensional Hopf algebra.

If we reformulate Lemma 1.2 for the left Haar functional $\varphi$, we get that the map $a \rightarrow \varphi(\cdot a)$ from $A$ to $A^{\prime}$ is bijective. This brings us naturally to the following definition.

1.4. Definition. Let $A$ be any algebra over $\mathbb{C}$ and $f$ a linear functional. We call $f$ faithful if $f(b a)=0$ for all $b$ implies $a=0$.

In principle there is a left and a right notion. However, because here $A$ is finitedimensional, one implies the other. Indeed, assume that $f$ is faithful. So the map $a \rightarrow f(\cdot a)$ from $A$ to $A^{\prime}$ is injective. Then it is also surjective. If $b \neq 0$ there exists $g \in A^{\prime}$ such that $g(b) \neq 0$. But $g$ has the form $f(\cdot a)$ for some $a$. Hence if $f(b a)=0$ for all $a$, then $b=0$.

It is clear that there exist algebras without a faithful functional (e.g. if $a b=0$ for all $a$ and $b$ ). We have seen however that, when $A$ is a finite-dimensional Hopf algebra, the Haar functionals are always faithful.

We have the following characterization of the left Haar functional on $A^{\prime}$.

1.5. Proposition. Let $A$ be a finite-dimensional Hopf algebra and $f$ any faithful linear functional on $A$. There is a unique element $h$ in A given by $f(a h)=\epsilon(a)$ for all $a$. This element satisfies ah $=\epsilon(a) h$ for all $a$ in $A$.

Proof. Because $f$ is faithful, any linear functional is given by a translation of $f$. If we apply this to $\epsilon$ we get the element $h$. It is also unique. 
For all $a, b \in A$ we have

$$
\begin{aligned}
f(a \epsilon(b) h) & =\epsilon(b) f(a h)=\epsilon(b) \epsilon(a) \\
& =\epsilon(a b)=f(a b h) .
\end{aligned}
$$

By uniqueness $b h=\epsilon(b) h$.

So we see that, given a faithful functional, we get the existence and uniqueness of the left Haar functional on $A^{\prime}$ very easily. And if we use Lemma 1.2, we find that this functional is faithful on $A^{\prime}$ and the result above, applied to $A$, gives the unique left Haar functional on $A$.

From the above results, we can also give a short argument for the fact that $S$ must be injective for a finite-dimensional Hopf algebra. Suppose $S a=0$. Then

$$
S a=S a(\iota \otimes \varphi) \Delta(h)=(\iota \otimes \varphi)((1 \otimes a) \Delta(h))=0 .
$$

Hence $\varphi(a \cdot)=0$. Then $\varphi(a b)=0$ for all $b$. By the surjectivity of the above map, $\omega(a)=0$ for all $\omega$ and $a=0$.

By symmetry we also have a unique right Haar functional. The one can be obtained from the other by means of the antipode. Considering these two Haar functionals, we can distinguish two different cases :

1.6. Proposition. If $A$ is a finite-dimensional Hopf algebra then one of the following statements in true :

i) One can choose a left Haar functional $\varphi$ such that $\varphi(1)=1$. Then $\varphi$ is invariant under $S$ and it is also a right Haar functional.

ii) For any left Haar functional $\varphi$ and right Haar functional $\psi$ we have $\varphi(1)=$ $\psi(1)=0$.

Proof. If $\varphi$ and $\psi$ are left and right Haar functionals, then $(\psi \otimes \varphi) \Delta(a)=\psi(a) \varphi(1)$ $=\psi(1) \varphi(a)$ for all $a$. Then the result follows easily.

In the next section we will see that we are automatically in the first case when the underlying algebra $A$ is a finite-dimensional $\mathrm{C}^{*}$-algebra. In section 3 we will give an example where the second alternative is true and where left and right Haar functionals are different.

\section{Finite-dimensional Hopf $\mathrm{C}^{*}$-Algebras}

If $A$ is a ${ }^{*}$-algebra and $f$ a positive linear functional then $f$ satisfies the CauchySchwarz inequality

$$
\left|f\left(b^{*} a\right)\right|^{2} \leq f\left(b^{*} b\right) f\left(a^{*} a\right) .
$$

It follows that $f$ is faithful (in the sense of Definition 1.4) if and only if $f\left(a^{*} a\right)=0$ implies $a=0$. This is the usual notion of faithfulness for positive linear functions on a *algebra. The finite-dimensional algebra $A$ has a faithful positive linear functional if and only if it has a faithful *-representation on a finite-dimensional Hilbert space. This means that $A$ is a finite-dimensional $\mathrm{C}^{*}$-algebra.

In particular, if $(A, \Delta)$ is a finite-dimensional Hopf algebra and if $A$ is a $\mathrm{C}^{*}$ algebra we can use the argument in the previous section to obtain the Haar functionals.

We will now assume that $A$ is a finite-dimensional $\mathrm{C}^{*}$-algebra further in this section and see what can be said more in this case. 
2.1. Proposition. There exists an element $h$ such that $\epsilon(h)=1$ satisfying ah $=$ $\epsilon(a) h$ for all $a$. This element is unique and also satisfies $h a=\epsilon(a) h$ for all $a$ and $h^{2}=h=h^{*}$.

Proof. Suppose that $h$ is non-zero and satisfies $a h=\epsilon(a) h$ for all $a$. Then $h^{*} h=$ $\epsilon\left(h^{*}\right) h=\overline{\epsilon(h)} h$. If $\epsilon(h)=0$ then $h^{*} h=0$ and $h=0$. Hence we can assume $\epsilon(h)=1$ so that $h^{*} h=h$. Then $h$ is self-adjoint. By taking adjoints, we find that also $h a=\epsilon(a) h$ when $a$ is in $A$.

This shows that we are in the first situation of Proposition 1.6 for the algebra $A^{\prime}$.

From the uniqueness one can easily show that the Haar functional is positive in this case (using the decomposition of linear functionals). This will imply that also for $A$ we have a Haar functional which is left and right invariant. But in this case one can prove a lot more. The result is due to Woronowicz (see [9], [10]). We give a short proof here.

2.2. Theorem. The left and right Haar functionals on $A$ coincide and they are positive traces.

Proof. Let $h$ be the element obtained in the previous proposition. We have seen in the proof of Lemma 1.2 that $(1 \otimes a) \Delta(h)=(S a \otimes 1) \Delta(h)$ for all $a \in A$. Similarly we have $\Delta(h)(a \otimes 1)=\Delta(h)(1 \otimes S a)$.

Now consider the trace $\operatorname{Tr}$ on $A$, normalized such that minimal projections get trace 1 . This exists because $A$ is a finite-dimensional $\mathrm{C}^{*}$-algebra. Define $K=$ $(\operatorname{Tr} \otimes \iota) \Delta(h)$. This will give a positive element in $A$. We claim that it is invertible. If it is not, there exists a non-zero positive linear functional $\omega$ on $A$ such that $\omega(K)=0$. So $\operatorname{Tr}((\iota \otimes \omega) \Delta(h))=0$ and this is impossible because $(\iota \otimes \omega) \Delta(h)$ is positive and non-zero.

For any $a$ we have

$$
\begin{aligned}
a K & =(\operatorname{Tr} \otimes \iota)((1 \otimes a) \Delta(h)) \\
& =(\operatorname{Tr} \otimes \iota)((S a \otimes 1) \Delta(h)) \\
& =(\operatorname{Tr} \otimes \iota)(\Delta(h)(S a \otimes 1)) \\
& =(\operatorname{Tr} \otimes \iota)\left(\Delta(h)\left(1 \otimes S^{2} a\right)\right) \\
& =K S^{2} a .
\end{aligned}
$$

Now let $\varphi$ be a left Haar functional on $A$. We have seen that we can assume $\varphi(h)=1$. Because $a K=(\operatorname{Tr} \otimes \iota)((S a \otimes 1) \Delta(h))$ we will get $\varphi(a K)=\operatorname{Tr}(S a)$. We must have that $\operatorname{Tr}(S a)=\operatorname{Tr}(a)$ because $S$ is an anti-homomorphism and the uniqueness of the trace. Hence $\varphi(a)=\operatorname{Tr}\left(a K^{-1}\right)$ for all $a$. It follows that $\varphi$ is positive and $\varphi(1) \neq 0$. Then left and right functionals coincide. This proves the first statement in the theorem and the positivity.

Since left and right Haar functionals are the same, we must have $\varphi(S a)=\varphi(a)$ for all $a$. This implies $S K=K$. If we apply $S$ to the equation $a K=K S^{2} a$ we get $K S a=S^{3} a K$ for all $a$ and $K a=S^{2} a K$. So $K^{2} a=a K^{2}$. By positivity of $K$, we also have $K a=a K$ for all $a$. This implies that $\varphi$ is a trace.

Note that we get the same formula for the left Haar functional as in [8] for discrete quantum groups. However, here we see that $S^{2} a=a$ for all $a$ because $K$ commutes with $a$ and implements $S^{2}$. 
The formula obtained in the proof of 1.1 , with $b=1$ and dualized gives

$$
\varphi(x)=\sum_{i} f_{i}\left(x S^{2} a_{i}\right)=\sum_{i} f_{i}\left(x a_{i}\right) .
$$

This is indeed a trace because

$$
\begin{aligned}
\varphi(x y) & =\sum_{i} f_{i}\left(x y a_{i}\right) \\
& =\sum_{i, j} f_{i}\left(x a_{j}\right) f_{j}\left(y a_{i}\right)=\sum_{j} f_{j}\left(y x a_{j}\right) .
\end{aligned}
$$

We see that the trace property of $\varphi$ is connected with the fact that $S^{2}=\iota$.

One can show that $A^{\prime}$ is again a $\mathrm{C}^{*}$-algebra. In a way, this is a surprising fact. We prove this by more or less standard arguments using the positivity of the Haar functional in the next proposition.

2.3. Proposition. Let $\varphi$ be a positive left Haar functional on A. Consider the G.N.S. representation associated with $\varphi$. On the associated Hilbert space there is also a faithful ${ }^{*}$-representation of $A^{\prime}$.

Proof. Let $A^{\prime}$ act on $A$ in the usual way by $\pi(f)(a)=(\iota \otimes f) \Delta(a)$. It is easy to see that $\pi(f g)=\pi(f) \pi(g)$. This action is also faithful. Indeed, if $\pi(f)=0$ then $(\iota \otimes f) \Delta(a)=0$ for all $a$. Then apply $\epsilon$ to obtain that $f(a)=0$ for all $a$ and hence $f=0$.

We will show that

$$
\varphi\left(a^{*}(\pi(f) b)\right)=\varphi\left(\left(\pi\left(f^{*}\right) a\right)^{*} b\right)
$$

for all $a$ and $b$ in $A$. This will complete the proof of the proposition.

Now

$$
\begin{aligned}
\varphi\left(a^{*}(\pi(f) b)\right) & =\sum_{(b)} \varphi\left(a^{*} b_{(1)}\right) f\left(b_{(2)}\right) \\
& =\sum_{(a),(b)} \varphi\left(a_{(1)}^{*} b_{(1)}\right) f\left(S^{-1} a_{(3)}^{*} a_{(2)}^{*} b_{(2)}\right) \\
& =\sum_{(a)}\left(\varphi \cdot f\left(S^{-1} a_{(2)}^{*} \cdot\right)\right)\left(a_{(1)}^{*} b\right) \\
& =\sum_{(a)} \varphi\left(a_{(1)}^{*} b\right) f\left(S^{-1} a_{(2)}^{*}\right) \\
& =\sum_{(a)} \varphi\left(a_{(1)}^{*} b\right) f^{*}\left(a_{(2)}\right)^{-} \\
& =\varphi\left(\left(\pi\left(f^{*}\right) a\right)^{*} b\right) .
\end{aligned}
$$

\section{An example}

In this section we treat an example of a finite-dimensional Hopf algebra where left and right invariant Haar functionals are different. We will also illustrate some other aspects of the theory.

Let $\lambda \in \mathbb{C}$ and $|\lambda|=1$. Consider the ${ }^{*}$-algebra $A$ over $\mathbb{C}$ with identity, generated by two self-adjoint elements $a$ and $b$ such that $a$ is invertible and $a b=\lambda b a$. This 
algebra can be made into a Hopf *-algebra if we define $\Delta$ by $\Delta(a)=a \otimes a$ and $\Delta(b)=a \otimes b+b \otimes a^{-1}$ (see e.g. [7]). This Hopf *-algebra can also be paired with itself. If $z \in \mathbb{C}$ and $z^{2}=\lambda$, such a pairing is given by

$$
\begin{aligned}
\langle a, a\rangle & =z, & \langle a, b\rangle & =0, \\
\langle b, a\rangle & =0, & \langle b, b\rangle & =i \bar{z} .
\end{aligned}
$$

If $\lambda$ is not a root of unity, the pairing is non-degenerate. If $\lambda$ is a root of unity, the pairing is degenerate and gives rise to quotients that are (in most cases) finitedimensional. Again see [7]. We will consider these finite-dimensional cases here, but we will obtain them directly. We will also be a little more general.

So let $\lambda \in \mathbb{C}$ and assume that $\lambda$ is a root of unity. Let $n$ be the smallest non-zero element in $\mathbb{N}$ such that $\lambda^{2 n}=1$. Assume $n \geq 2$ (so we exclude the cases $\lambda=1$ and $\lambda=-1)$. Also let $m$ be any non-zero element in $\mathbb{N}$ such that $\lambda^{m}=1$.

3.1. Definition. Let $A$ be the ${ }^{*}$-algebra over $\mathbb{C}$ with identity generated by selfadjoint elements $a$ and $b$ such that $a^{m}=1, b^{n}=0$ and $a b=\lambda b a$.

3.2. Proposition. The elements $\left\{a^{p} b^{q} \mid p=0,1, \ldots, m-1\right.$ and $\left.q=0,1, \ldots, n-1\right\}$ form a basis for $A$.

Proof. It is clear that these elements span $A$ as a vector space. We show that they are linearly independent by constructing a representation. Let $V=\mathbb{C}^{m} \otimes \mathbb{C}^{n}$ and consider the standard basis $\left\{e_{p} \otimes f_{q} \mid p=0,1, \ldots, m-1\right.$ and $\left.q=0,1, \ldots, n-1\right\}$. Let $z=\exp \frac{2 \pi i}{m}$ and define linear operators $\pi(a)$ and $\pi(b)$ on $V$ by

$$
\begin{aligned}
& \pi(a) e_{p} \otimes f_{q}=z^{p} \lambda^{-q} e_{p} \otimes f_{q}, \\
& \pi(b) e_{p} \otimes f_{q}= \begin{cases}e_{p} \otimes f_{q-1} & \text { if } q \neq 0, \\
0 & \text { if } q=0 .\end{cases}
\end{aligned}
$$

It is easy to check that $\pi(a)^{m}=1, \pi(b)^{n}=0$ and $\pi(a) \pi(b)=\lambda \pi(b) \pi(a)$. So we obtain a representation of $A$ (not a ${ }^{*}$-representation). It is clear that in this representation, the elements $a^{p} b^{q}$ are linearly independent.

3.3. Proposition. $A$ is a Hopf ${ }^{*}$-algebra if we define $\Delta(a)=a \otimes a$ and $\Delta(b)=$ $a \otimes b+b \otimes a^{-1}$. The counit $\epsilon$ is given by $\epsilon(a)=1$ and $\epsilon(b)=0$ and the antipode $S$ is given by $S a=a^{-1}$ and $S b=-\lambda^{-1} b$.

Proof. To prove the existence of $\Delta$, given by the above formulas, we essentially only have to show that $\left(a \otimes b+b \otimes a^{-1}\right)^{n}=0$ in $A \otimes A$.

Now, by induction one can show that, if $q=0,1, \ldots, n$, we have

$$
\left(a \otimes b+b \otimes a^{-1}\right)^{q}=\sum_{k=0}^{q} C_{k}^{q} a^{k} b^{q-k} \otimes a^{-(q-k)} b^{k}
$$

where $C_{0}^{q}=C_{q}^{q}=1$ and

$$
C_{k}^{q}=\frac{r_{1} \ldots r_{q}}{r_{1} \ldots r_{k} r_{1} \ldots r_{q-k}}
$$

if $k=1,2, \ldots, q-1$ and where

$$
r_{k}=\frac{\lambda^{k}-\lambda^{-k}}{\lambda-\lambda^{-1}}
$$

if $k=1,2, \ldots, n$ (see e.g. [4] for similar calculations). 
Note that $\lambda \neq 1$ and $\lambda \neq-1$, so that the formula for $r_{k}$ makes sense, and that $r_{k} \neq 0$ for all $k=1, \ldots, n-1$ so that the formula for $C_{k}^{q}$ makes sense.

Because $\lambda^{2 n}=1$ we have $r_{n}=0$ and $C_{k}^{n}=0$ when $k=1,2, \ldots, n-1$. Hence

$$
\left(a \otimes b+b \otimes a^{-1}\right)^{n}=a^{n} \otimes b^{n}+b^{n} \otimes a^{-n} .
$$

This is 0 because $b^{n}=0$ in $A$.

The rest of the proof is standard. The counit $\epsilon$ is well-defined on $A$ because the pair $(1,0)$ satisfies the same relations as the pair $(a, b)$ and the antipode is welldefined because the pair $\left(a^{-1},-\lambda^{-1} b\right)$ satisfies the same relations for the opposite product. See e.g. [7] for more complete arguments.

Let us now try to obtain some information about $A^{\prime}$. We do this by considering two special elements and by looking at the action of $A^{\prime}$ on $A$.

3.4. Definition. Let $z$ be any non-zero complex number. Define elements $c$ and $d$ in $A^{\prime}$ by

$$
\begin{aligned}
& \left\langle c, a^{p} b^{q}\right\rangle= \begin{cases}z^{p} & \text { if } q=0, \\
0 & \text { if } q \neq 0,\end{cases} \\
& \left\langle d, a^{p} b^{q}\right\rangle= \begin{cases}1 & \text { if } q=1, \\
0 & \text { if } q \neq 1 .\end{cases}
\end{aligned}
$$

We know that $A^{\prime}$ acts on $A$ by means of the formula $\langle x, \pi(y) z\rangle=\langle x y, z\rangle$ whenever $x, y \in A^{\prime}$ and $z \in A$. In the next proposition we obtain $\pi(c)$ and $\pi(d)$.

3.5. Proposition. For all $p$ and $q$ we have

$$
\begin{aligned}
& \pi(c) a^{p} b^{q}=z^{p-q} a^{p} b^{q}, \\
& \pi(d) a^{p} b^{q}= \begin{cases}r_{q} a^{p+1} b^{q-1}, & q \neq 0, \\
0, & q=0 .\end{cases}
\end{aligned}
$$

Proof. For any $x \in A^{\prime}$ and $p, q$ we have

$$
\begin{aligned}
\left\langle x c, a^{p} b^{q}\right\rangle & =\left\langle x \otimes c,\left(a^{p} \otimes a^{p}\right)\left(a \otimes b+b \otimes a^{-1}\right)^{q}\right\rangle \\
& =\sum_{k=0}^{q} C_{k}^{q}\left\langle x, a^{p} a^{k} b^{q-k}\right\rangle\left\langle c, a^{p} a^{-(q-k)} b^{k}\right\rangle \\
& =C_{0}^{q}\left\langle x, a^{p} b^{q}\right\rangle z^{p-q}
\end{aligned}
$$

so that $\pi(c) a^{p} b^{q}=z^{p-q} a^{p} b^{q}$.

Similarly, for any $x \in A^{\prime}$ and $p, q$ we have

$$
\begin{aligned}
\left\langle x d, a^{p} b^{q}\right\rangle & =\left\langle x \otimes d,\left(a^{p} \otimes a^{p}\right)\left(a \otimes b+b \otimes a^{-1}\right)^{q}\right\rangle \\
& =\sum_{k=0}^{q} C_{k}^{q}\left\langle x, a^{p} a^{k} b^{q-k}\right\rangle\left\langle d, a^{p} a^{-(q-k)} b^{k}\right\rangle \\
& =C_{1}^{q}\left\langle x, a^{p+1} b^{q-1}\right\rangle
\end{aligned}
$$

if $q \neq 0$. We get 0 if $q=0$. So $\pi(d) a^{p} b^{q}=r_{q} a^{p+1} b^{q-1}$ if $q \neq 0$ and $\pi(d) a^{p} b^{q}=0$ if $q=0$. 
We clearly have $\pi(d)^{n}=0$. So also $d^{n}=0$ because $\pi$ is faithful. But we have more.

3.6. Proposition. $c d=z^{2} d c$.

Proof. If $q \neq 0$ we have

$$
\begin{aligned}
\pi(c) \pi(d) a^{p} b^{q} & =r_{q} \pi(c) a^{p+1} b^{q-1} \\
& =r_{q} z^{p+1-q+1} a^{p+1} b^{q-1} \\
& =z^{2} z^{p-q} \pi(d) a^{p} b^{q} \\
& =z^{2} \pi(d) \pi(c) a^{p} b^{q} .
\end{aligned}
$$

If $q=0$ we have $\pi(c) \pi(d) a^{p} b^{q}=0$ and $\pi(d) \pi(c) a^{p} b^{q}=0$. Again because $\pi$ is faithful we get the desired result.

If $z$ is also a root of unity, some power of $c$ will also be equal to 1 . In fact, if $z^{2}=\lambda$ and $z^{m}=1$ we essentially get the same algebra as the one we started with. This is related with the self-duality that was obtained in ([7]). Also remark that we get here almost the same representation as in Proposition 3.2.

Let us now look at the left and right invariant elements in $A$.

\subsection{Proposition. Let}

$$
h=\sum_{p=1}^{m} a^{p} b^{n-1} \quad \text { and } \quad k=\sum_{p=1}^{m} b^{n-1} a^{p} ;
$$

then $x h=\epsilon(x) h$ and $k x=\epsilon(x) k$ for all $x \in A$.

Proof. Because $a^{m}=1$ we have

$$
a \sum_{\ell=1}^{m} a^{\ell}=\sum_{\ell=1}^{m} a^{\ell}
$$

and therefore $a h=h=\epsilon(a) h$. Further

$$
b h=\sum_{\ell=1}^{m} b a^{\ell} b^{n-1}=\sum_{\ell=1}^{m} \lambda^{-\ell} a^{\ell} b^{n}=0
$$

and $b h=\epsilon(b) h$ because $\epsilon(b)=0$. Because $\epsilon$ is a homomorphism, we then have $x h=\epsilon(x) h$ for all $x \in A$. Similar arguments are used to show that $k x=\epsilon(x) k$ for all $x \in A$.

It is easy to verify here that $h$ and $k$ are the unique elements satisfying these equalities. Note that $h \neq k$. Indeed, this algebra is not a $\mathrm{C}^{*}$-algebra because $b^{n}=0$ and $b$ is self-adjoint. In a $\mathrm{C}^{*}$-algebra this would imply $b=0$.

Let us now verify the faithfulness of these elements. If we let $c$ act on $h$ we find

$$
\pi(c) h=\sum_{p=0}^{m-1} z^{p} z^{-n+1} a^{p} b^{n-1} .
$$

So, if we choose $z=\exp \frac{2 \pi i}{m}$ as we did before, by repeated action of $\pi(c)$ we will obtain the space spanned by $\left\{a^{p} b^{n-1} \mid p=0, \ldots, m-1\right\}$. Then repeated action of $\pi(d)$ will give us all elements $a^{p} b^{q}$. This shows the faithfulness of $h$. Similarly for $k$.

We can also find easily the unique left and right Haar functionals on $A$. 
3.8. Proposition. Define $\varphi$ and $\psi$ on $A$ by

$$
\begin{aligned}
& \varphi\left(a^{p} b^{q}\right)= \begin{cases}1 & \text { if } a^{p}=a^{-(n-1)} \text { and } q=n-1, \\
0 & \text { otherwise }\end{cases} \\
& \psi\left(a^{p} b^{q}\right)=\left\{\begin{array}{ll}
1 & \text { if } a^{p}=a^{n-1} \\
0 & \text { otherwise }
\end{array} \text { and } q=n-1,\right.
\end{aligned}
$$

then, $\varphi$ is a left and $\psi$ a right Haar functional on $A$.

Proof. Let $p$ be any integer and let $q=0,1, \ldots, n-1$. Then

$$
(\iota \otimes \varphi) \Delta\left(a^{p} b^{q}\right)=\sum_{k=0}^{q} C_{k}^{q} a^{p+k} b^{q-k} \varphi\left(a^{p-q+k} b^{k}\right) .
$$

The right-hand side will be 0 except if $k=n-1$ and $a^{p-q+k}=a^{-(n-1)}$. This can only happen if $q=n-1$ and $a^{p}=a^{-(n-1)}$. In that case, we get precisely 1 . Hence $(\iota \otimes \varphi) \Delta\left(a^{p} b^{q}\right)=\varphi\left(a^{p} b^{q}\right)$ for all $p, q$.

Similarly

$$
(\psi \otimes \iota) \Delta\left(a^{p} b^{q}\right)=\sum_{k=0}^{q} C_{k}^{q} \psi\left(a^{p+k} b^{q-k}\right) a^{p-q+k} b^{k} .
$$

The right-hand side will be 0 except if $q-k=n-1$ and $a^{p+k}=a^{n-1}$. This will only happen if $k=0, q=n-1$ and $a^{p}=a^{n-1}$. Also here, we get 1 in that case. And again $(\psi \otimes \iota) \Delta\left(a^{p} b^{q}\right)=\psi\left(a^{p} b^{q}\right)$ for all $p, q$.

It is clear that also here, $\varphi$ and $\psi$ are different. Moreover, the formula for $\psi$ can be obtained from the formula for $\varphi$ by applying the antipode $S$.

\section{ACKNOWLEDGEMENT}

I want to thank my colleagues at the university of Oslo, where I spent the summer in 1992 for their warm hospitality. I would especially like to thank Professor E. Alfsen for the many discussions we had during that summer on the Haar measureboth for the usual locally compact groups and the quantum groups.

\section{REFERENCES}

[1] E. Abe, Hopf Algebras, Cambridge University Press, 1977. MR 83a:16010

[2] S. Baaj \& G. Skandalis, Unitaires Multiplicatifs et dualité pour les Produits Croisés de $C^{*}$ Algèbres. Ann. Scient. Ec. Norm. Sup., 4ème sŕie, 26 (1993), 425-488. MR 94e:46127

[3] E.G. Effros \& Z.-J. Ruan, Discrete Quantum Groups I. The Haar Measure, Int. J. Math. 5 (1994), 681-723. MR 95j:46089

[4] T. Koornwinder, Representations of the twisted $S U(2)$ quantum group and some qhypergeometric orthogonal polynomials, Proc. Koninklijke Nederlandse Akademie Wetenschappen, Series A 92 (1989), 97-117. MR 90h:17016

[5] S. Sakai, $C^{*}$-algebras and $W^{*}$-algebras, Springer Verlag (Berlin), 1971. MR 56:1082

[6] M.E. Sweedler, Hopf Algebras, Mathematical Lecture Notes Series, Benjamin (New York), 1969. MR 40:5705

[7] A. Van Daele, Dual Pairs of Hopf*-algebras, Lecture notes (second version), Bull. London. Math. Soc. 25 (1993), 209-230. MR 94c: 16053

[8] A. Van Daele, Discrete Quantum Groups, J. of Algebra 180 (1996), 431-444. MR 97a:16076 
[9] S.L. Woronowicz, Compact Matrix Pseudogroups, Comm. Math. Physics 111 (1987), 613665. MR 88m:46079

[10] S.L. Woronowicz, Compact quantum groups, Preprint University of Warsaw (1992).

Department of Mathematics, K.U. Leuven, Celestijnenlaan 200B, B-3001 Heverlee, BELGIUM

E-mail address: alfons.vandaele@wis.kuleuven.ac.be 